\title{
Hautpflege im Intimbereich: Weniger ist oft mehr
}

\author{
Eine spezielle Intimpflege ist bei gesunden Frauen nicht \\ zwingend. Anders verhält es sich aber, wenn die Intim- \\ haut trocken, gereizt oder empfindlich ist. Dann braucht \\ die Haut die richtige Pflege.
}

Frau Dr. Widmer, worin besteht der Unterschied zwischen Intimpflege und Intimhygiene?

Dr. Widmer: Intimhygiene ist die Reinigung der Intimregion (bei der Frau Vulva und Anus) von Körpersäften und Gerüchen. Intimpflege ist die Pflege der Intimhaut, wenn diese einer besonderen Pflege bedarf, sei sie trocken, gereizt, gerötet oder einfach empfindlich.

Was kann die Intimhaut belasten?

Dr. Widmer: Geschlechtsverkehr, chloriertes Wasser in Schwimmbädern, übertriebenes Waschen (vor allem mit alkalischen Seifen) sowie das Tragen enger Jeans und Strings können die Intimhaut belasten und Irritationen auslösen. Gelegentlich führen auch eine Intimrasur und Haarentfernungsmittel zu Reizungen im Intimbereich.

Worauf sollen die Frauen bei der Intimpflege verzichten? Dr. Widmer: Auf übertriebene Hygiene, vor allem auf vorsorgliche Scheidenspülungen.

Sollen auch gesunde Frauen Intimpflege betreiben - und wenn ja, wie?

Dr. Widmer: Nein, das ist nicht nötig, aber sie dürfen natürlich ihren Intimbereich pflegen. Eine tägliche Intimpflege fördert schließlich den sinnlichen Kontakt zum eigenen Genitale.

Worauf müssen ältere Patientinnen bei der Intimpflege achten? Dr. Widmer: Ältere Patientinnen haben oft eine trockene Haut. Je trockener die Haut ist, desto fetter sollte die Grundpflege sein.

Auf welche Inhaltsstoffe ist bei Waschlotionen für den Intimbereich speziell zu achten?

Dr. Widmer: Sanfte Waschlotionen schützen den Hautsäuremantel und sind vorzugsweise rückfettend.

Welche Inhaltsstoffe sind eher zu meiden und welche können zu Allergien oder Irritationen führen?

Dr. Widmer: Das lässt sich pauschal nicht beantworten. Die Reaktionen auf die Inhaltsstoffe sind zu individuell. Darum: ausprobieren.

Welche Cremes und Öle sind besonders geeignet?

Dr. Widmer: Ich empfehle Produkte mit hochwertigen Trägersubstanzen und Inhaltsstoffen, am liebsten pflanzliche Produkte.

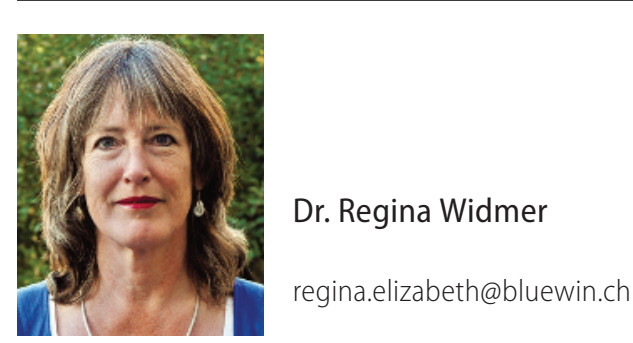

Worauf sollten Frauen bei der Intimpflege verzichten? Dr. Widmer: Auf zu viel Pflege. Es braucht meist nur wenig Öl oder Creme.

Welche Ursachen kann vaginale Trockenheit haben? Dr. Widmer: Das Alter und zu häufiges Duschen, Baden oder Waschen - übrigens auch Waschen nur mit Wasser. Wasser trocknet grundsätzlich aus.

Bei welchen Patientinnen sind östrogenhaltige Cremes und östrogenhaltige Zäpfchen indiziert?

Dr. Widmer: Ich verschreibe sie Frauen mit einer dünnen Vaginalhaut und wenn Frauen beim Geschlechtsverkehr, im Wochenbett oder in und nach den Wechseljahren über eine trockene Scheide klagen, insbesondere wenn noch zusätzliche Beschwerden wie eine leicht irritierbare Harnröhre und Blase mit verstärktem Harndrang hinzukommt. Als erstes rate ich aber meinen Patientinnen nur, ihren Intimbereich gut zu fetten. Klingen die Symptome dann innerhalb von einer bis zwei Wochen nicht wesentlich ab, verschreibe ich östrogenhaltige Cremes oder Vaginalzäpfchen. Diese wenden die Frauen zu Behandlungsbeginn etwa zweimal pro Woche an. Später, wenn die Beschwerden abgenommen haben, empfehle ich, Creme oder Zäpfchen noch einmal wöchentlich zu nehmen. Als Ergänzung verschreibe ich noch ein pflanzliches Mittel, das die Patientinnen an den anderen Wochentagen nach Bedarf anwenden können.

Wann spricht man von einem Lichen sclerosus et atrophicus und worauf muss der Arzt achten, um diese Erkrankung nicht zu übersehen? Dr. Widmer: Zu Beginn eines Lichen sclerosus et atrophicus ist die Haut oft gerötet und weiter nicht auffällig. In diesem Stadium sollten Ärztinnen und Ärzte die Krankheit bereits erkennen und nicht erst, wenn die Intimhaut pergamentartig dünn und weisslich ist und sich die inneren Venuslippen und der Scheideneingang verkleinert haben. Für eine eindeutige Diagnose braucht es eine Hautgewebeanalyse von einem pathologischen Institut.

Interview: Verena Vermeulen und Claudia Benetti 